\title{
Alteração da terapia antirretroviral: inefetividade ou insegurança?
}

\author{
Change of antirretroviral therapy: ineffectiveness or insecurity? \\ Cambiar terapia antirretroviral: inefectividad o inseguridad?
}

Recebido: 03/01/2022 | Revisado: 07/01/2022 | Aceito: 18/01/2022 | Publicado: 20/01/2022

\author{
Diego Pinheiro Mesquita \\ ORCID: https://orcid.org/0000-0002-3197-3791 \\ Universidade de Fortaleza, Brasil \\ E-mail: diego.pinheiro.m@icloud.com \\ Andressa Silva Araújo \\ ORCID: https://orcid.org/0000-0002-9522-9304 \\ Universidade de Fortaleza, Brasil \\ E-mail: andressasilva.as46@gmail.com \\ Erika Maria Siqueira Mourão \\ ORCID: https://orcid.org/0000-0001-6703-6612 \\ Hospital Distrital Gonzaga Mota Messejana, Brasil \\ E-mail: erikamouraoj@gmail.com \\ Geysa Aguiar Romeu \\ ORCID: https://orcid.org/0000-0002-4899-1072 \\ Universidade de Fortaleza, Brasil \\ Hospital Distrital Gonzaga Mota Messejana, Brasil \\ E-mail: geysa@unifor.br
}

\begin{abstract}
Resumo
O objetivo do estudo foi analisar as alterações da terapia antirretroviral de pacientes atendidos em uma unidade dispensadora de medicamentos de Fortaleza, Ceará. Realizou-se estudo observacional, documental, descritivo, retrospectivo, com abordagem quantitativa, no período de janeiro a maio de 2020. Incluíram-se todos os portadores de HIV/AIDS, cadastrados na unidade dispensadora de medicamentos, com idade igual ou superior a 18 anos que realizaram mudanças na terapia antirretroviral no período de janeiro a dezembro de 2019. Excluíram-se os usuários que mudaram de unidade durante esse período. Predominaram pessoas do sexo masculino, faixa etária entre 31 a 40 anos, pardos, solteiros, com escolaridade correspondente do ensino médio ao ensino superior, naturais e procedentes de Fortaleza, com tempo de diagnóstico entre 1 e 5 anos. Foram registradas 24 combinações diferentes de terapia antirretroviral, sendo a associação mais utilizada com tenofovir, lamivudina e efavirenz. Dos motivos que levaram à mudança de terapia antirretroviral, prevaleceram a incidência ou risco de reação adversa ao medicamento, início ou fim da gestação e facilitação da adesão ao tratamento. O efavirenz foi o principal medicamento envolvido nas suspeitas de reação adversa, seguido pelo tenofovir. Conclui-se que os dados obtidos auxiliaram na compreensão do perfil das pessoas vivendo com HIV de uma Unidade Dispensadora de Medicamentos de Fortaleza e as causas para mudança da terapia antirretroviral.
\end{abstract}

Palavras-chave: Efeitos colaterais e reações adversas relacionados a medicamentos; Síndrome de Imunodeficiência adquirida; Infecções por HIV; Fármacos Anti-HIV; Assistência ao paciente.

\begin{abstract}
The aim of the study was to analyze the changes in antiretroviral therapy in patients treated at a medicine dispensing unit in Fortaleza, Ceará. An observational, documentary, descriptive, retrospective study with a quantitative approach was carried out from January to May 2020. All HIV/AIDS patients, registered in the drug dispensing unit, aged 18 or over were included. years that made changes in antiretroviral therapy from January to December 2019. Users who changed units during this period were excluded. There was a predominance of males, aged between 31 and 40 years, brown, single, with education corresponding to high school to higher education, natives and coming from Fortaleza, with diagnosis time between 1 and 5 years. Twenty-four different combinations of antiretroviral therapy were recorded, the most used association being tenofovir, lamivudine and efavirenz. Of the reasons that led to the change in antiretroviral therapy, the incidence or risk of adverse drug reaction, beginning or end of pregnancy and facilitating adherence to treatment prevailed. Efavirenz was the main drug involved in suspected adverse reactions, followed by tenofovir. It is concluded that the data obtained helped to understand the profile of people living with HIV in a Medicine Dispensing Unit in Fortaleza and the causes for changing antiretroviral therapy.
\end{abstract}

Keywords: Drug-related side effects and adverse reactions; Acquired immunodeficiency syndrome; HIV infections; Anti-HIV Agents; Patient care. 


\begin{abstract}
Resumen
El objetivo del estudio fue analizar los cambios en la terapia antirretroviral en pacientes atendidos en una unidad de dispensación de medicamentos en Fortaleza, Ceará. Se realizó un estudio observacional, documental, descriptivo, retrospectivo con abordaje cuantitativo de enero a mayo de 2020. Se incluyeron todos los pacientes con VIH/SIDA, registrados en la unidad de dispensación de medicamentos, de 18 años o más. Años que realizaron cambios en la terapia antirretroviral desde enero a diciembre de 2019. Se excluyeron los usuarios que cambiaron de unidad durante este período. Predominó el sexo masculino, con edades entre 31 y 40 años, morenos, solteros, con educación correspondiente a bachillerato a nivel superior, nativos y provenientes de Fortaleza, con tiempo de diagnóstico entre 1 y 5 años. Se registraron veinticuatro combinaciones diferentes de terapia antirretroviral, siendo la asociación más utilizada tenofovir, lamivudina y efavirenz. De las razones que motivaron el cambio en la terapia antirretroviral, prevalecieron la incidencia o riesgo de reacción adversa al medicamento, inicio o finalización del embarazo y facilitación de la adherencia al tratamiento. Efavirenz fue el principal fármaco implicado en las sospechas de reacciones adversas, seguido de tenofovir. Se concluye que los datos obtenidos ayudaron a comprender el perfil de las personas que viven con el VIH en una Unidad de Dispensación de Medicamentos en Fortaleza y las causas del cambio de terapia antirretroviral.
\end{abstract}

Palabras clave: Efectos colaterales y reacciones adversas relacionados con medicamentos; Síndrome de inmunodeficiencia adquirida; Infecciones por VIH; Fármacos anti-VIH; Atención al paciente.

\title{
1. Introdução
}

A síndrome da imunodeficiência adquirida (AIDS) começou a ser observada no início da década de 1980, nos Estados Unidos da América, em razão do surgimento de pacientes que apresentavam um declínio da imunidade celular, tendo como resultado a morte por infecções oportunistas e/ou neoplasias atípicas. Na busca pelo entendimento dos recorrentes casos, logo após o desenvolvimento de testes sorológicos, notou-se que a diminuição da imunidade de pessoas vivendo com HIV/AIDS (PVHIV) correspondia à redução da contagem das células linfócitos T CD4+ em função do aumento da carga viral em um processo crônico. Algumas pessoas tinham sua imunidade diminuída de maneira abrupta, enquanto outras, a infecção seguia seu curso de maneira mais lenta, porém, sempre culminando na morte dos infectados (Salomão, 2017).

O vírus da imunodeficiência humana (HIV) infecta e destrói preferencialmente linfócitos TCD4, resultando na perda da imunidade celular e alta probabilidade de que o hospedeiro desenvolva infecções oportunistas. Outras células, tais como macrófagos e monócitos, que possuem proteína CD4 em sua superfície podem também ser atingidas (Levinson, 2016).

A partir da compreensão das fases da replicação do vírus identificam-se os alvos terapêuticos inibidores específicos do ciclo de replicação viral, como por exemplo, os inibidores de transcriptase reversa análogos e não análogos de nucleosídeo, inibidores de proteases, inibidores de entrada (fusão e co-receptor) e inibidores de integrase (Salomão, 2017).

Segundo o Departamento de Informática do SUS (DATASUS) do ano de 1980 até o ano de 2019, mais de 960 mil pessoas foram infectadas e diagnosticadas com HIV no Brasil. A precisão desses dados é importante para se traçar estratégias de controle e prevenção da infecção, portanto, os profissionais da saúde devem notificar, por meio do Sistema de Informação de Agravos de Notificação (SINAN), todos os casos novos de infecção por HIV (Ministério da Saúde [MS], 2018).

Neste contexto, o Brasil assumiu junto à Organização das Nações Unidas (ONU), o compromisso de combater e reverter a tendência atual de propagação do HIV. O país tem como meta para o ano de 2020, alcançar o percentual de $90 \%$ das pessoas com HIV tenham diagnóstico; também é almejado ampliar o acesso ao tratamento para que $90 \%$ destas, realizem a terapia antirretroviral (TARV); e que, das pessoas em tratamento, 90\% tenham a carga viral indetectável. Portanto, a atenção básica cumpre papel fundamental, ampliando o acesso ao diagnóstico e tratamento das PVHIV (MS, 2018).

Visando alcançar as metas previstas, o Sistema Único de Saúde (SUS) oferta gratuitamente os exames de diagnóstico e monitoramento da infecção (Camargo et al., 2014), medicamentos utilizados na TARV, bem como os medicamentos voltados para o tratamento das infecções oportunistas (MS, 2018).

Portanto, a pessoa diagnosticada portadora do HIV, assistida pelo médico será avaliada e participará da pactuação do tratamento inicial, em seguida é direcionado para uma Unidade Dispensadora de Medicamentos (UDM) antirretrovirais (ARV) 
onde, além de receber os medicamentos, é acompanhada pelo serviço atenção farmacêutica, que é fundamental, uma vez que a partir dessa prática pode-se identificar e prevenir problemas relacionados ao medicamento e evitar a má adesão ao tratamento (Prado et al., 2016).

Apesar dos medicamentos serem bastante eficazes, o sucesso da terapia não depende apenas do potencial farmacológico, diversos motivos podem influenciar a falha da terapia, muitas vezes relacionados à falta de adesão ao tratamento antirretroviral (Ferreira et al., 2010). Tais falhas na adesão ao tratamento podem estar relacionadas aos sentimentos do indivíduo em relação à infecção, condições de vida, motivações e obstáculos ao tratamento. Outros fatores sociodemográficos, tais como, idade, raça, sexo, renda e escolaridade; e clínicos, associado com efeitos colaterais de medicações, estresse psicológico e o uso de outras substâncias além da terapia antirretroviral, são apontados também por afetar negativamente a adesão terapêutica (Fernándes et al., 2018).

A não adesão ou baixa adesão a TARV trazem problemas tanto individuais quanto geral, pois, implica na alteração do esquema terapêutico, onde possivelmente será um tratamento mais complexo envolvendo maior número de comprimidos, além de favorecer a resistência viral, impactando negativamente políticas públicas voltadas a infecção por HIV e aumentando a probabilidade de infecções oportunistas (Silva, et al., 2015).

Neste contexto, esse estudo objetivou buscar informações sobre as alterações na TARV confrontando com suas causas, por meio da caracterização do perfil sócio demográfico e clínico dos pacientes portadores de HIV/AIDS e assim, colaborar para o entendimento das nuances que permeiam a TARV e seus protocolos de manejo.

\section{Metodologia}

Realizou-se estudo documental, descritivo, retrospectivo, com abordagem quantitativa (Gil, 2002), no período de janeiro a maio de 2020, em uma Unidade Dispensadora de Medicamentos (UDM) antirretrovirais de Fortaleza, Ceará. O Serviço de Atendimento Especializado (SAE) para pacientes com HIV/AIDS conta com uma equipe multidisciplinar constituída por médicos, enfermeiros, farmacêuticos, assistentes sociais e psicólogos. A UDM está incorporada à farmácia hospitalar de um hospital de referência materno-infantil da cidade de Fortaleza e oferece serviço de atenção farmacêutica (Prefeitura de Fortaleza, 2018).

Incluíam-se todos pacientes portadores de HIV/AIDS cadastrados no serviço de farmácia, com idade igual ou superior a 18 anos que realizaram alteração da TARV no período de janeiro a dezembro de 2019. Excluíram-se os usuários que mudaram de unidade durante esse período.

A população do estudo correspondeu a 1.099 pacientes cadastrados na farmácia. Destes, 146 tiveram sua terapia alterada, 10 pacientes foram excluídos. Portanto, a análise foi feita a partir dos dados de 136 pacientes. Foram estudadas variáveis sócio demográficas, terapias antirretrovirais utilizadas e motivo da mudança da TARV.

Coletaram-se os dados dos prontuários dos pacientes, das fichas de cadastro na farmácia e do Sistema de Controle Logístico de Medicamentos (SICLOM) do Ministério da Saúde. Esse sistema permite que o Departamento de Vigilância, Prevenção e Controle das IST/HIV/AIDS e das Hepatites Virais se mantenha atualizado em relação ao fornecimento de medicamentos aos pacientes em TARV, nas várias regiões do país. As informações são utilizadas para o controle do estoque e na distribuição dos antirretrovirais, assim como para a obtenção de informações clínico-laboratoriais dos pacientes e uso de diferentes esquemas terapêuticos (Ministério da Saúde, 2018).

As informações foram transcritas para formulário de coleta de dados pré-validado. As informações foram transcritas para formulário de coleta de dados pré-validado. Os dados foram analisados quantitativamente pelo programa Epiinfo versão 7.2.3.1. As variáveis numéricas serão descritas como média e desvio padrão e as variáveis categóricas sob forma de referência absoluta (n) e relativa (\%). O programa Microsoft Excel 2021 foi utilizado para elaboração de gráficos e tabelas. 
A realização desse estudo foi autorizada pelo responsável legal da UDM e aprovado no Comitê de Ética em Pesquisa do Hospital sob número do parecer 3.027.219. Atendeu às Normas e Diretrizes para pesquisas com seres humanos estabelecidas na Resolução n. 466/2012 do Conselho Nacional de Saúde/MS.

\section{Resultados}

Prevaleceram pessoas do sexo masculino $(55,9 \% ; n=76)$. A razão entre os sexos foi de 1,3 casos em homens para cada caso em mulheres. A idade dos pacientes variou entre 21 e 78 anos (média 42,9 \pm 12,2 anos). Outras características predominantes foram: pardos $(49,3 \%$; $n=67)$; solteiros $(39,0 \% ; n=53)$; com escolaridade correspondente do ensino médio ao ensino superior (39,7\%; $\mathrm{n}=54)$; com tempo de diagnóstico entre 1 e 5 anos $(46,3 \%$; $\mathrm{n}=63)$; naturais (49,3\%; $\mathrm{n}=67)$ e procedentes $(83,1 \% ; n=113)$ de Fortaleza (Tabela 1$)$.

Tabela 1: Distribuição das pessoas vivendo com HIV/Aids segundo características sócio demográficas (2019).

\begin{tabular}{|c|c|c|}
\hline Variáveis & $\mathrm{n}^{\mathbf{0}}$ & $\%$ \\
\hline \multicolumn{3}{|l|}{ Sexo } \\
\hline Feminino & 60 & 44,1 \\
\hline Masculino & 76 & 55,9 \\
\hline \multicolumn{3}{|l|}{ Faixa etária (anos) } \\
\hline 18 a 30 & 26 & 19,1 \\
\hline 31 a 40 & 39 & 28,7 \\
\hline 41 a 50 & 37 & 27,2 \\
\hline 51 a 60 & 23 & 16,9 \\
\hline$>60$ & 11 & 8,1 \\
\hline \multicolumn{3}{|l|}{ Estado civil } \\
\hline Solteiro & 53 & 39,0 \\
\hline Casado & 17 & 12,5 \\
\hline Separado/Divorciado & 3 & 2,2 \\
\hline União estável & 13 & 9,6 \\
\hline Viúvo & 4 & 2,9 \\
\hline Não informado & 46 & 33,8 \\
\hline \multicolumn{3}{|l|}{ Raça } \\
\hline Branca & 24 & 17,6 \\
\hline Parda & 67 & 49,3 \\
\hline Preta & 9 & 6,6 \\
\hline Não informado & 35 & 25,8 \\
\hline \multicolumn{3}{|l|}{ Escolaridade (anos) } \\
\hline Nenhuma & 3 & 2,2 \\
\hline 1 a 3 & 8 & 5,9 \\
\hline 4 a 7 & 25 & 18,4 \\
\hline 8 a 11 & 30 & 22,1 \\
\hline$>12$ & 24 & 17,7 \\
\hline Não informado & 46 & 33,8 \\
\hline \multicolumn{3}{|l|}{ Naturalidade } \\
\hline Fortaleza & 67 & 49,3 \\
\hline Interior do Ceará & 56 & 41,2 \\
\hline Outros estados & 13 & 9,6 \\
\hline \multicolumn{3}{|l|}{ Procedência } \\
\hline Fortaleza & 113 & 83,1 \\
\hline Interior do Ceará & 22 & 16,2 \\
\hline Outros estados & 1 & 0,7 \\
\hline \multicolumn{3}{|c|}{ Tempo de diagnóstico (anos) } \\
\hline$<1$ & 4 & 2,9 \\
\hline 1 a 5 & 63 & 46,3 \\
\hline 6 a 10 & 59 & 43,4 \\
\hline$>10$ & 10 & 7,4 \\
\hline
\end{tabular}

Fonte: Autores. 
Durante a análise, foram registradas 24 combinações diferentes de TARV. A associação mais utilizada foi o "3 em 1", composto pelo tenofovir (TDF) + lamivudina (3TC) + efavirenz (EFZ) $(24,8 \%$; n=36). As demais combinações são apresentadas na Figura 1.

Figura 1: Terapias antirretrovirais utilizadas pelos pacientes antes da alteração do tratamento farmacológico (2019).

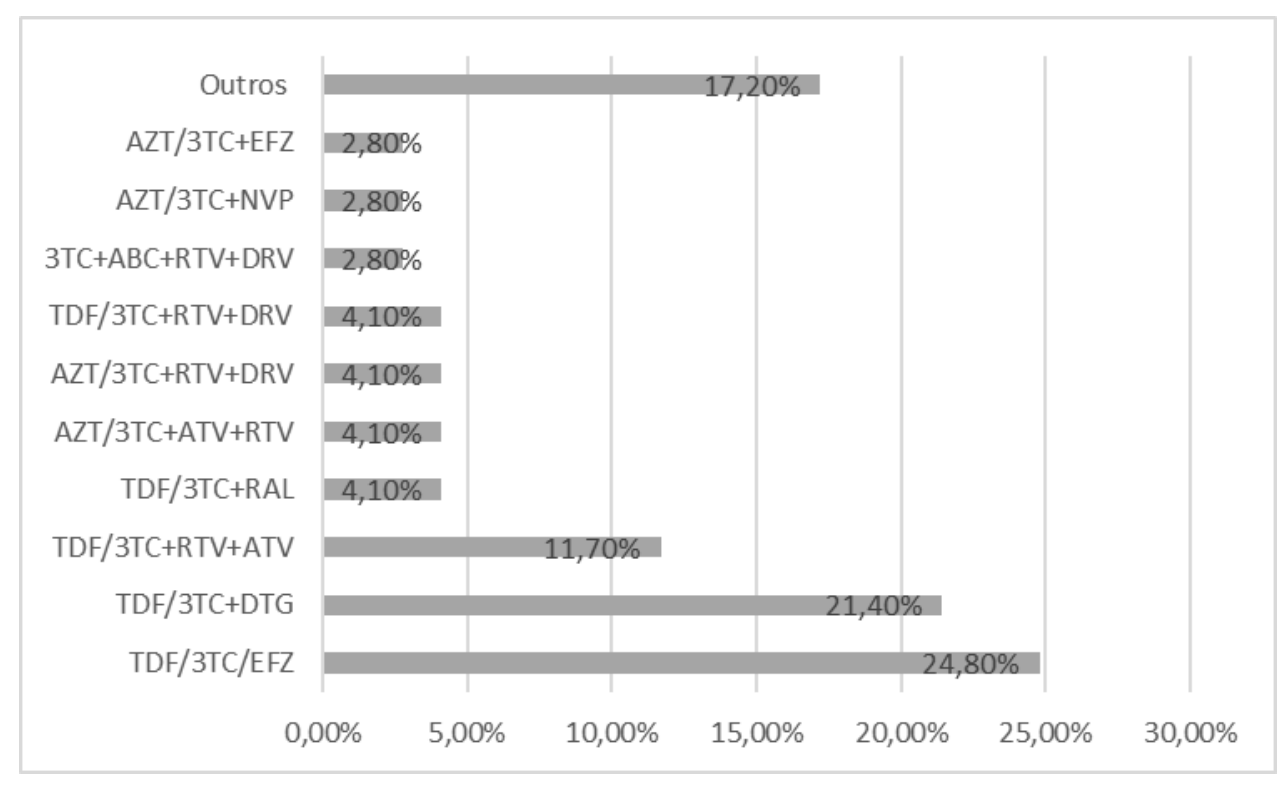

Descrição: 3TC (lamivudina), ABC (abacavir), ATV (atazanavir), AZT (zidovudina), DGT (dolutegravir), DRV (darunavir), EFZ (efavirenz), NVP (nevirapina), RAL (raltegravir), RTV (ritonavir), TDF (tenofovir). Fonte: Autores.

A Tabela 2 retrata os motivos que levaram à mudança de TARV. Destacaram-se a incidência ou risco de reação adversa a medicamento (RAM) $(64,2 \%$; $=88)$, início ou fim da gestação $(8,0 \%$; $=11)$, falha viral detectado por genotipagem $(2,2 \% ; n=3)$ e intenção de facilitar a adesão ao tratamento $(11,7 \% ; n=16)$ desses, 7 pacientes tiveram a terapia reduzida para uma combinação com 3 ou menos ARV.

É importante relatar que houve pacientes que mudaram a terapia mais de uma vez nesse período, sendo assim, nesses casos, foram registradas mais de uma resposta por paciente.

Tabela 2: Motivos para mudança da terapia antirretroviral (2019).

\begin{tabular}{lll}
\hline Motivos para mudança da TARV & $\mathbf{N}$ & $\mathbf{\%}$ \\
\hline Incidência ou risco de RAM & 88 & 64,2 \\
Falha terapêutica & 4 & 2,9 \\
Co-infecção por tuberculose & 4 & 2,9 \\
Facilitar adesão à TARV & 16 & 11,7 \\
Início ou fim de gestação & 11 & 8,0 \\
Falta do medicamento & 2 & 1,5 \\
Interação medicamentosa & 4 & 2,9 \\
Retorno após abandono de TARV & 5 & 3,6 \\
Genotipagem & 3 & 2,2 \\
\hline
\end{tabular}

Descrição: RAM: reação adversa a medicamentos; TARV: terapia antirretroviral. Fonte: Autores. 
A Figura 2, evidencia que o EFZ $(29,30 \%$; n=27) foi o principal medicamento envolvido nos casos suspeitos de reação adversa, seguido pelo TDF $(22,80 \%$; $=21)$ e ATV $(12 \%$; $=11)$. Das 88 suspeitas de RAM, identificou-se o tipo de reação em 81 casos (Quadro 1).

Figura 2: Antirretrovirais suspeitos de causar reações adversas ou envolvidos no risco de reações (2019).

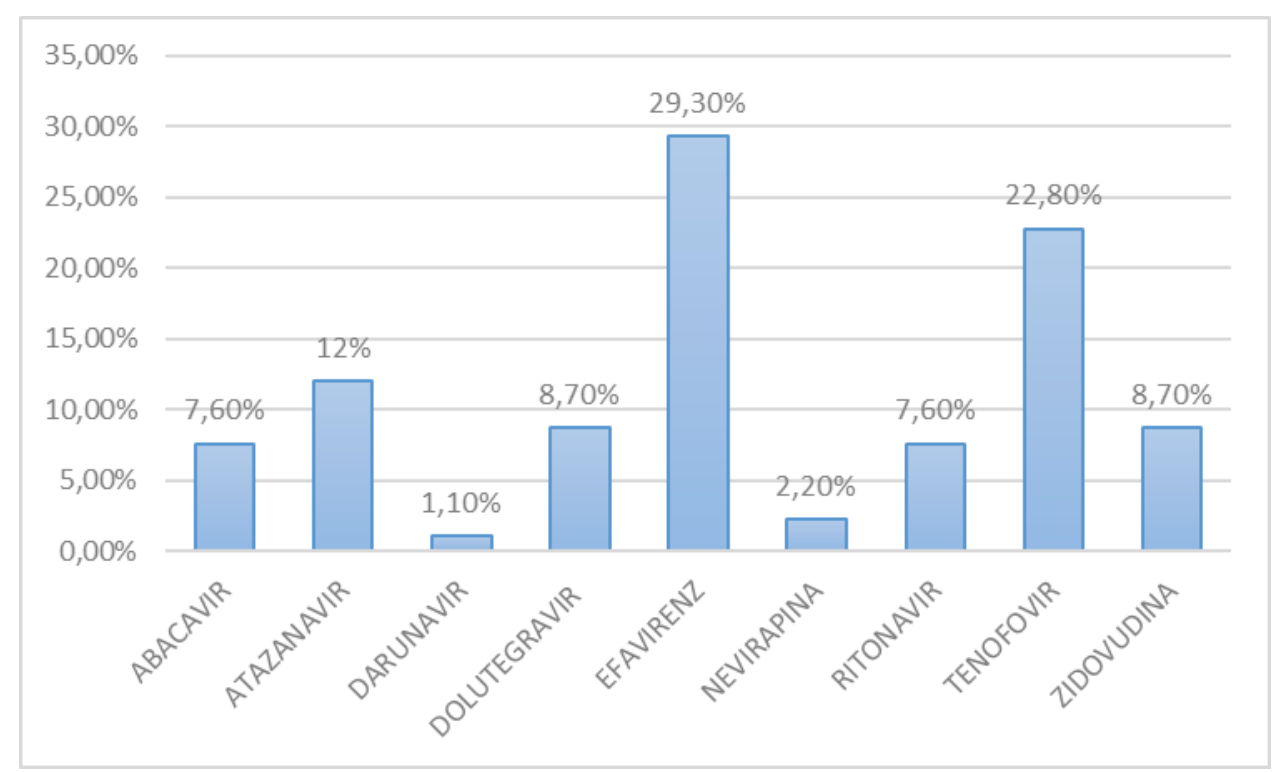

Fonte: Autores.

O Quadro 1, descreve as principais suspeitas de reações adversas, relacionando com a Figura 2 pelos antirretrovirais que mais causarão tais reações, como o Efavirenz e o Tenofovir. Podemos destacar que as principais reações descritas, forão náuseas e icterícia.

Quadro 1: Suspeitas de reações adversas aos antirretrovirais (2019).

\begin{tabular}{|l|l|}
\hline Reação Adversa ao Medicamento & Antirretroviral \\
\hline $\begin{array}{l}\text { Diminuição do paladar e olfato; dificuldade de cicatrização óssea; náuseas; lesão } \\
\text { renal; dor nas articulações }\end{array}$ & Tenofovir \\
\hline Distúrbio alimentar; potencial risco cardiovascular & Abacavir \\
\hline Hepatotoxicidade; icterícia & Ritonavir \\
\hline Icterícia; manchas na pele & Atazanavir \\
\hline Náuseas & Zidovudina \\
\hline Náuseas & Nevirapina \\
\hline $\begin{array}{l}\text { Tonturas; sonolência; neurotoxicidade; ansiedade; diarreia; insônia; irritabilidade; } \\
\text { estresse }\end{array}$ & Efavirenz \\
\hline
\end{tabular}

Fonte: Autores.

\section{Discussão}

O boletim epidemiológico da Secretaria de Vigilância em Saúde do Ministério da Saúde mostra que, em 2018, a detecção de AIDS entre homens foi de 25,2 casos a cada 100.000 habitantes. Entre as mulheres, observou-se tendência de queda dessa taxa nos últimos dez anos, que passou de 17,0 para 10,5 casos/100.000 habitantes entre 2008 e 2018 , revelando 
um decréscimo de 38,2\%. A razão dos sexos apresenta diferenças regionais. No Nordeste, a razão de sexos foi de 2,3 casos em homens para cada caso em mulheres (Secretaria de Vigilância em Saúde \& Ministério da Saúde, 2019).

A razão de sexos encontrado neste estudo foi menor quando comparado à razão nacional de 2018 e menor também ao encontrado por Santos, Silva \& Soares (2010) em pesquisa realizada em Goiânia, onde obtiveram uma razão homem/mulher de 2,76 casos e já alertavam sobre a feminização da AIDS em função do comportamento sócio sexual da população e da vulnerabilidade biológica, tendência essa observada no estado e no país (Santos et al., 2010). No entanto, um estudo realizado na cidade de Fortaleza, em 2015, mostrou uma razão de 4,05 casos em homens para cada mulher (Reis, 2014).

O predomínio de solteiros remete à preocupação do entendimento sobre a infecção, suas consequências e formas de transmissão. A relação entre pessoas vivendo com HIV mostra a existência de parceiros sorodiscordantes e que, muitas vezes, a sorologia do parceiro é desconhecida. A utilização do preservativo em todas as relações sexuais ainda não é uma prática realizada de forma assídua e consistente, principalmente entre parceiros sexuais discordantes, o que demostra a necessidade de implementar melhorias às práticas preventivas nos serviços de atendimento especializados (Oliveira et al., 2020).

O nível de estudo do paciente é um fator importante para melhor adesão ao tratamento (Carvalho et al., 2019). Posteriormente, este aspecto reflete na alteração da TARV pois, o paciente que possui estudo formal é menos suscetível a mudanças no seu esquema terapêutico (Alene et al., 2019). Outro estudo também identificou pacientes com nível de escolaridade bom, encontrando-se entre o ensino médio e ensino superior (Santos, Silva \& Soares, 2010). Pessoas com baixa escolaridade, podem se beneficiar com as orientações sobre o uso adequado e seguro dos ARV, o que ressalta a importância do serviço de atenção farmacêutica oferecido pela UDM, visto que, o usuário da TARV se favorece de um tratamento com nomes dos medicamentos complexos. Em pesquisa realizada nesta mesma UDM, revelou um nível de satisfação dos usuários bastante satisfatório em relação ao serviço de atenção farmacêutica (Romeu et al., 2009).

Em relação à TARV, entre os anos de 2013 a 2017 a combinação de primeira escolha para pessoas infectadas pelo HIV sem outros agravos era TDF+3TC+EFZ (Ministério da Saúde [MS], 2013), combinação essa que predominou nos casos estudados. A segunda combinação mais prescrita foi TDF+3TC+DTG, que está alinhada com a primeira linha de tratamento estabelecida pelo Ministério da Saúde a partir de janeiro de 2017 (Ministério da Saúde, 2018). Verificou-se, portanto, que as prescrições de antirretrovirais obedeceram aos padrões sugeridos nos protocolos clínicos disponibilizados pelo Ministério da Saúde.

Diversos fatores influenciam a durabilidade da TARV e vão além dos medicamentos envolvidos. Um estudo realizado nos EUA com dados de 8 locais de tratamentos para PVHIV relata que variáveis como gênero, idade e cor da pele podem influencia significativamente a continuidade do tratamento, o mesmo estudo também demonstra que o tratamento antirretroviral baseado em classes medicamentosas como inibidores da transcriptase reversa não análogos a nucleotídeos; inibidores da integrase proporcionam uma maior continuidade do tratamento (Eaton el al., 2018).

O presente estudo assim como diversos outros apontam que a principal causa para alteração na TARV é a ocorrência de reação adversa, além de ser considerada uma das principais causas para interrupção do tratamento, ameaçando a meta pactuada entre o Brasil e a ONU de alcançar, em 2020, 90\% das pessoas infectadas com o HIV fazendo a TARV (Ministério da Saúde, 2018; Vázquez et al., 2004; Padua et al., 2017).

Em meio às causas de mudança terapêutica, também se destacou a facilitação da adesão ao tratamento, onde pode envolver mais de um comprimido diferente com uma ou mais de uma tomada ao dia, detectando que a maioria das alterações dentro desse aspecto, foram realizadas por meio da redução do número de fármacos ARV. Sabe-se que a simplificação da TARV para tratamentos que requerem menos tomadas ao dia favorece a adesão, e os resultados se mostram ainda mais positivos quando se sugerem horários fixos para a tomada, incluindo-o em alguma parte da rotina do usuário (Carvalho et a., 
2019). Neste estudo, foi observado que na maior parte dos casos que tiveram alterações da TARV para melhorar a adesão, foi favorecida com a combinação de 3 ou menos ARV, como também relatado em outro estudo (Silva et al., 2017).

Outro fator importante na mudança da TARV é norteado pelo período gestacional, onde o uso do DTG é contraindicado no Brasil, pois apesar de não haver evidências relacionando ele, assim como outros inibidores de integrase com a toxicidade fetal, as informações relatadas em literatura ainda são insuficientes. Uma alternativa ao DTG seria o EFV, que apresenta segurança comprovada na gestação por não apresentar aumento de defeitos congênitos quando utilizado durante gravidez, todavia por seus efeitos neurológicos, fazem com que se prefira o raltegravir (RAL) pela segurança e efetividade, já que em comparação com o EFV apresenta maior taxa de queda da carga viral, sendo assim esquema de primeira linha para gestantes é TDF+3TC+RAL, devendo ser feito a substituição para o DTG após o fim da gestação (Ministério da Saúde [MS], 2019).

A falha terapêutica, menos evidenciada neste estudo para justificar a alteração da TARV, pode ser identificada por meio de alterações na contagem de linfócitos TCD4+ e carga viral, entre outros métodos (Reis, 2014). Para corrigi-la, são necessárias intervenções baseando-se nos protocolos clínicos para o manejo da terapia antirretroviral. A mudança deve ser, preferencialmente, guiada por teste de genotipagem, a depender da situação do paciente ou dos medicamentos envolvidos (Ministério da Saúde, 2018).

Com relação aos medicamentos envolvidos nas reações adversas, a 3TC destacou-se positivamente por sua segurança, haja vista que mais esteve presente nas TARV e não apresentou reações adversas no estudo, fato que corrobora com os resultados já descritos (Reis, 2014). O DRV e DTG também se mostraram seguros quanto à ocorrência de reações adversas.

Enquanto o EFZ ganhou destaque por ser o medicamento mais apontado como responsável por causar reações. A ele foram atribuídos diversos efeitos de cunho neurotóxico. O uso do TDF foi relacionado à lesão renal e diminuição da capacidade de cicatrização óssea. Portanto, em casos de fratura óssea optou-se pela retirada desse ARV tendo em vista a diminuição da densidade óssea causada por esse medicamento (Venter et al., 2018; Ministério da Saúde, 2018).

A investigação do motivo de troca de apenas do primeiro esquema ARV pode ser apontada como uma limitação deste estudo, podendo assim subestimar os motivos desencadeados por outros fármacos utilizados posteriormente. Além disso, os ARV foram analisados isoladamente, ignorando as possíveis reações adversas ocasionadas por medicamentos utilizados para outras doenças crônicas ou infecções oportunistas.

\section{Conclusão}

Os dados obtidos auxiliaram na compreensão do perfil das PVHIV de uma UDM de Fortaleza e as causas para mudança da TARV. O esquema terapêutico mais prescrito, composto por tenofovir, lamivudina e efavirenz, evidenciou que os prescritores seguiram o protocolo do Ministério da Saúde vigente na época que antecedeu o estudo e contribuiu para a maior incidência de reação adversa causada pelo efavirenz, seguido pelo tenofovir.

O estudo denota a importância da percepção dos efeitos adversos dos medicamentos envolvidos na terapia, visto que foi o principal motivo para mudança de tratamento. Informações não abordadas como: efetividade dos tratamentos realizados, informações sobre o conhecimento da população infectada com HIV da sua condição clínica, levando em conta a importância da adesão à TARV, formas de prevenção, entre outros aspectos sugerem a necessidade em dar continuidade ao estudo.

\section{Referências}

Alene, M., Awoke, T., Yenit, M. K., Tsegaye, A. T., Yismaw, L. \& Yeshambel, R. (2019). Second-line antiretroviral therapy regimen change among adults living with HIV in Amhara region: a multi-centered retrospective follow-up study. BMC Res Notes, 12(407). 1-6. https://bmcresnotes.biomedcentral.com/articles/10.1186/s13104-019-4429-3\#citeas 
Camargo, B.V., Bousfield, A. B. S., Giacomozzi, A. I. \& Koelzer, L. P. (2014). Representações sociais adesão ao tratamento anti-retroviral. Liberabit, 20(2). 229-238. http://www.scielo.org.pe/pdf/liber/v20n2/a04v20n2.pdf

Carvalho, P. P., Barroso, S. M., Coelho, H. C. \& Penaforte, F. R. O. (2019). Fatores associados à adesão à Terapia Antirretroviral em adultos: revisão integrativa de literatura. Ciência \& Saúde Coletiva, 24(7). 2543-2555. https://www.scielo.br/pdf/csc/v24n7/1413-8123-csc-24-07-2543.pdf

Departamento de Informática do SUS - DATASUS. Informações de Saúde, Casos de HIV: banco de dados. http://www2.datasus.gov.br/DATASUS/index.php?area=0203\&id=6930

Eaton, E. F., Tamhane, A., Davy-Mendez, T., Mathews, W.C., Moore, R. D., Saag, M. S. \& Mugavero, M. J. (2018). Trends in antiretroviral therapy prescription,durability and modification: new drugs, more changes, but less failure. HHS Public Access, 32(3). 347-35. https://pubmed.ncbi.nlm.nih.gov/29194118/

Fernándes, C. A. S., Andrade, C. I. L. \& Jara, C. M. (2018). Adherencia al tratamiento antirretroviral como conducta promotora de salud en adultos mayores. Rev Cuid, 9(2). 2201-2214. https://pesquisa.bvsalud.org/portal/resource/pt/biblio-963438

Ferreira, R. C. S., Riffel, A. \& Sant'ana A. E. G. (2010). HIV: Mecanismo de replicação, alvos farmacológicos e inibição por produtos derivados de plantas. Química Nova, 33(8). 1743-1755. https://www.scielo.br/scielo.php?script=sci_abstract\&pid=S0100-40422010000800023\&lng=pt\&tlng=pt

Gil, A. C. (2002). Como elaborar projetos de pesquisa (4a ed.). Atlas.

Levinson W. (2016). Microbiologia Médica e Imunologia. Porto Alegre: Amgh $\quad$ Editora https://integrada.minhabiblioteca.com.br/\#/books/9788580555578/cfi/2!/4/4@0.00:35.1

Ministério da Saúde (2013). Protocolo Clínico e Diretrizes Terapêuticas para Manejo da Infecção pelo HIV em Adultos. Brasília: Ministério da Saúde. http://bvsms.saude.gov.br/bvs/publicacoes/protocolo_clinico_manejo_hiv_adultos.pdf

Ministério da Saúde (2018). Sistema de Controle Logístico de Medicamentos (SICLOM). Fortaleza. http://www.aids.gov.br/pt-br/gestores/sistemas-deinformacao/sistema-de-controle-logistico-de-medicamentos-siclom

Ministério da Saúde (2018). Protocolo Clínico e Diretrizes Terapêuticas para Manejo da Infecção pelo HIV em Adultos. Brasília: Ministério da Saúde. http://www.aids.gov.br/pt-br/pub/2013/protocolo-clinico-e-diretrizes-terapeuticas-para-manejo-da-infeccao-pelo-hiv-em-adultos

Ministério da Saúde (2019). Protocolo Clínico e Diretrizes Terapêuticas para Prevenção da Transmissão Vertical de HIV, Sífilis e Hepatites Virais. Brasília. http://www.aids.gov.br/pt-br/pub/2015/protocolo-clinico-e-diretrizes-terapeuticas-para-prevencao-da-transmissao-vertical-de-hiv

Oliveira, L. B., Costa, C. R. B., Ponte, P. S., Magalhães, R. L. B., Gir, E. \& Reis, R. K. (2020). Parceria sexual entre pessoas vivendo com HIV: gerenciando as diferenças sorológicas. Revista Eletrônica trimestral de Enfermaria, 19(58). 507-518. http://scielo.isciii.es/pdf/eg/v19n58/pt_1695-6141-eg-19-58-494.pdf

Pádua C. M., Braga L. P. \& Mendicino C. C. P. (2017). Adverse reactions to antiretroviral therapy: a prevalent concern. Rev Panamericana Salud Publica, 41(84). 1-5. https://scielosp.org/pdf/rpsp/2017.v41/e84/en

Prado, C. G., Podesta, M. H. C. M., Souza, L. P. T., Souza, W. A. \& Ferreira, E. B. (2016). Acompanhamento fármaco terapêutico de pacientes HIV positivos em uma unidade de dispensação de medicamentos antirretrovirais. Rev da Universidade Vale do Rio Verde, $14(2) . \quad 562-576$. http://periodicos.unincor.br/index.php/revistaunincor/article/view/2777

Prefeitura de Fortaleza. Canal Saúde: Serviços Ambulatoriais Especializados em HIV/AIDS. Fortaleza. https://saude.fortaleza.ce.gov.br/postosdesaude/2uncategorised/135-servicos-ambulatoriais-especializados-em-hiv-aids

Reis, H. P. L. C. (2014). Acompanhamento de pessoas com HIV sob terapia antirretroviral: adequação, aplicação e análise de indicadores clínicolaboratoriais, farmacoterapêuticos e humanísticos na atenção farmacêutica. (Tese de doutorado) - Faculdade de Farmácia, Odontologia e Enfermagem, Universidade Federal do Ceará, Fortaleza, CE, Brasil. http://www.repositorio.ufc.br/handle/riufc/10879

Romeu, G. A., Paiva, L. V. \& Moura Fé, M. M. (2009). Pharmaceutical care to pregnant women carrying human immunodeficiency virus. Brazilian Journal of Pharmaceutical Sciences, 45(3). 593-602. https://www.scielo.br/scielo.php?pid=S1984-82502009000300026\&script=sci_arttext

Salomão R. (2017). Síndrome da Imunodeficiência Adquirida. Infectologia: Bases Clinicas e Tratamento. Rio de Janeiro: Editora Guanabara Koogan Ltda. https://reumatologiapr.com.br/wp-content/uploads/2018/02/INFECTOLOGIA-BASES-CLINICAS-e-TRATAMENTO-2017.pdf

Santos, C. N. R., Silva, L. R. \& Soares, A. Q. (2010). Perfil epidemiológico dos pacientes em terapia antirretroviral em seguimento na Universidade Federal de Goiás. Revista Eletrônica de Farmácia, VII(3). 53-61. https://pdfs.semanticscholar.org/d64e/916f57062d834bca0d92141a9fb7b3b12fe4.pdf

Secretaria de Vigilância em Saúde \& Ministério da Saúde (2019). Boletim Epidemiológico: HIV/Aids. Brasília. http://www.aids.gov.br/ptbr/pub/2019/boletim-epidemiologico-de-hivaids-2019

Silva R. A. R., Nelson A. R. C., Duarte F. H. S., Prado N. C . C., Holanda J. R. R. \& Costa D. A. R. S. (2017). Avaliação da adesão à terapia antirretroviral em pacientes com Aids. Rev Fund Care Online, 9(1). 15-20. http://www.seer.unirio.br/index.php/cuidadofundamental/article/view/3736

Silva, J. A. G. S., Dourado, I., Brito, A. M. \& Silva, C. A. L. S. (2015). Fatores associados à não adesão aos antirretrovirais em adultos com AIDS nos seis primeiros meses da terapia em Salvador, Bahia, Brasil.Cadernos de Saúde Pública, 31(6). 1188-1198. https://www.scielo.br/scielo.php?script=sci_arttext\&pid=S0102-311X2015000601188

Vázquez, I. P., Framiñán, L. M., Fernández, K. P. \& Herranz, I. M. (2004). Cambio del tratamiento antirretroviral en el paciente VIH. Farmacia Hospitalaria, 28(1). 54-60. https://medes.com/publication/20408

Venter W. D. F., Fabian J. \& Feldman C. (2018). An overview of tenofovir and renal disease for the HIV-treating clinician. S Afr J HIV Med, 19(1). 1-8. http://www.scielo.org.za/pdf/sajhivmed/v19n1/20.pdf 